\title{
Acute interstitial nephritis related to immune checkpoint inhibitors
}

\author{
Julie Belliere ${ }^{1}$, Nicolas Meyer ${ }^{2,3,4}$, Julien Mazieres ${ }^{4,5}$, Sylvie Ollier ${ }^{6}$, Serge Boulinguez ${ }^{2}$, Audrey Delas ${ }^{7}$, \\ David Ribes ${ }^{1}$ and Stanislas Faguer ${ }^{\star}, 1,4,8$ \\ ${ }^{1}$ Département de Néphrologie et Transplantation d'organes, Centre de référence des maladies rénales rares, Hôpital Rangueil, \\ Toulouse, France; ${ }^{2}$ Service de Dermatologie-Oncologie, Institut Universitaire du Cancer de Toulouse-Oncopôle, Toulouse, \\ France; ${ }^{3}$ INSERM UMR 1037—CRCT, Toulouse, France; ${ }^{4}$ Université Toulouse-III, Toulouse, France; ${ }^{5}$ Service de Pneumologie, \\ Hôpital Larrey, Toulouse, France; ${ }^{6}$ Service de Médecine interne et Immunologie clinique, Institut Universitaire du Cancer de \\ Toulouse_Oncopôle, Toulouse, France; ' ${ }^{7}$ Laboratoire d'Anatomopathologie, Institut Universitaire du Cancer de Toulouse- \\ Oncopôle, Toulouse, France and ${ }^{8}$ INSERM U1048, Equipe 12 (Fibrose rénale), Institut des Maladies métaboliques et \\ cardiovasculaires, Toulouse, France
}

Background: Immune checkpoint inhibitors (anti-PD1 or anti-CTLA-4) are increasingly used in various cancers. Immune checkpoint inhibitors (ICI)-related renal disorders are poorly described (9 cases) and were only related to Ipilimumab.

Methods: Retrospective collection of clinical charts of all the patients admitted for renal disorders following ICI in the University Hospital of Toulouse (France).

Results: We report on adverse renal events that occurred in three patients treated with anti-PD1 (nivolumab or pembrolizumab) or anti-CTLA-4 (ipilimumab). Acute kidney injury occurred at 4-12 weeks after initiation of treatment, and harbored features of tubulo-interstitial nephritis (interstitial polymorphic inflammatory infiltrate with predominant CD3 + CD4 + T cells, associated with granuloma in one). Following withdrawal of $\mathrm{ICl}$ and steroid intake, estimated glomerular-filtration rate had improved in all patients.

Conclusions: These data suggest that all $\mathrm{ICl}$ can lead to acute interstitial nephritis, possibly related to the presence of autoreactive clonal T cells. We recommend that patients receiving $\mathrm{ICl}$ should undergo renal monitoring every 2 weeks for 3-6 months.

Monoclonal antibodies against the immune checkpoints, cytotoxic T-lymphocyte antigen-4 (CTLA-4), the programmed cell death protein 1 (PD-1), or its ligand (PD-L1) have demonstrated significant improvement in response rates, progression-free survival, and overall survival in melanoma, lung, and renal-cell carcinoma (Topalian et al, 2012). Ipilimumab, an anti CTLA-4 antibody (Bristol-Myers Squibb, New York, NY, USA) and nivolumab (Bristol-Myers Squibb, New York, NY, USA), and pembrolizumab (Merck Sharp and Dohme, New-York, NY, USA), both anti PD-1 inhibitors, now represent the standard-of-care frontline or second-line therapy in numerous advanced cancers. Immune check-point inhibitors (ICI), acting on immune system, don't share the toxicities of previously used classical chemotherapies (cytotoxic agents) like cytopenia, neuropathies, or hair loss. Immune-related adverse events are common, including colitis, pneumonitis, hepatitis, dermatitis, and hypophysitis (Voskens et al, 2013). Renal disorders are rare and first cases have very recently been reported (Izzedine et al, 2014; Cortazar et al, 2016; Shirali et al, 2016). Herein, we describe three patients that developed adverse renal events, respectively, after nivolumab, pembrolizumab, and ipilimumab treatment.

\section{PATIENTS AND METHODS}

Patients were recruited from the Nephrology Department of the University Hospital of Toulouse (South-West, France). According

*Correspondence: Dr S Faguer; E-mail: stanislas.faguer@inserm.fr

Received 20 May 2016; revised 21 September 2016; accepted 30 September 2016; published online 10 November 2016

(c) 2016 Cancer Research UK. All rights reserved 0007-0920/16 
the Declaration of Helsinki revised in 2004 and to the rules of the Ethical committee of the University Hospital of Toulouse, patients were informed of the collection of their clinical data and gave informed consent.

Renal and extra-renal involvements were recorded using standardised assessment of the hospital records. Glomerular filtration rate was estimated (eGFR) using the CKDEPI formula. Renal pathology consisted of light microscopy and immunostaining directed against IgG, IgA, IgM, kappa and lambda light-chains, and fibrinogen. Immunostainings against CD3 T-cell and CD20 B-cell markers were also performed.

\section{Patients}

Case 1. A 67-year-old woman with lung adenocarcinoma was treated with nivolumab. She was a never-smoker and had no significant comorbidities. Other treatments included omeprazole, bromazepam, fluoxetine, and levothyroxine. She had previously received three lines of chemotherapy, including platinum salt, and had no renal impairment. As shown in Figure 1, serum creatinine (SCr) was $78 \mu \mathrm{moll}^{-1}$ at baseline (day 0; estimated glomerularfiltration rate (eGFR) was $68 \mathrm{ml} \mathrm{min}^{-1} / 1.73 \mathrm{~m}^{2}$ ). Urinalysis showed no red or white cells, and was sterile. After the third infusion of nivolumab (day 34), the patient developed fatigue and
A

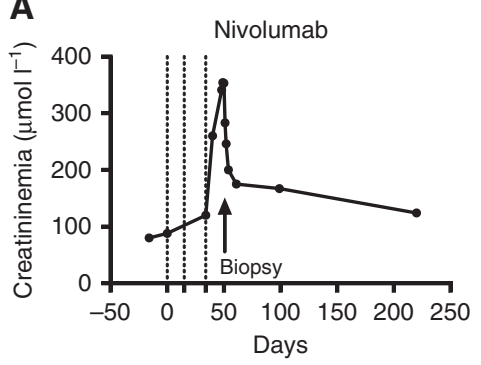

D

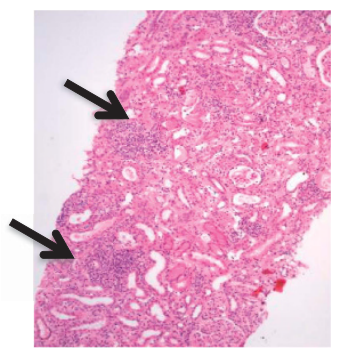

$\mathbf{F}$

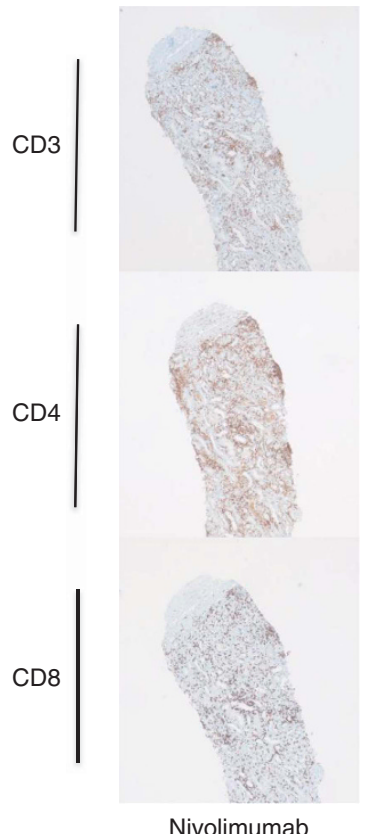

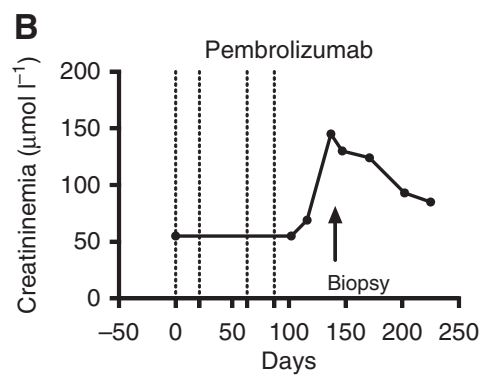

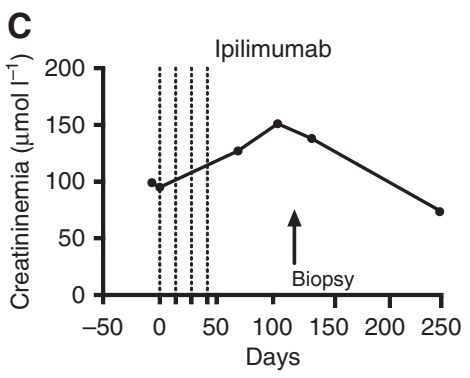

E

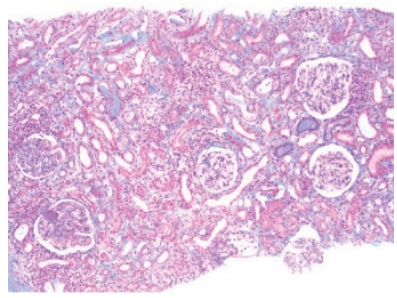

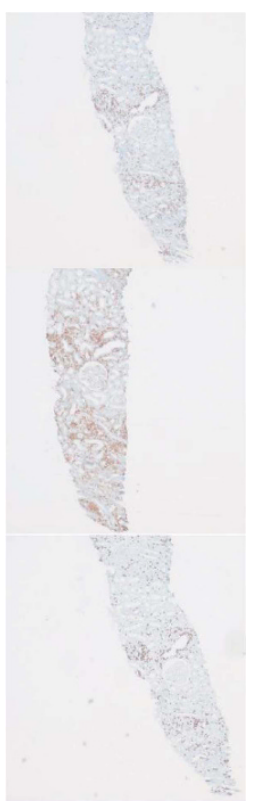

Pembrolizumab

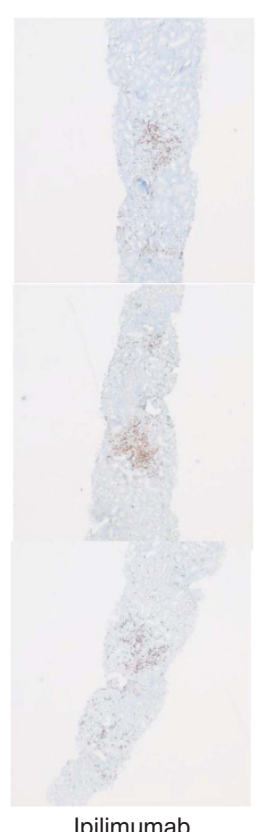

Ipilimumab

Figure 1. Interstitial nephritis following the use of immune checkpoint inhibitors. Serum creatinine is depicted according to the time that patients were treated with nivolumab (A), pembrolizumab (B), or ipilimumab (C). Each vertical line indicates one injection of an immune checkpoint inhibitor. The arrow represents a kidney biopsy. ( $\mathbf{D}$ and $\mathbf{E}$ ) Hematoxylin-eosin and Masson' trichrome staining of a kidney section (original magnification) revealed acute interstitial nephritis with lymphoplasmacytic cell infiltrate and acute tubular injuries. Arrows indicate granulomatosis aspect of the infiltrate. (F) Anti-CD3, CD4, and CD8 staining (original magnification x50). The interstitial inflammatory infiltrate was mainly positive for the CD3 CD4 T-cell marker. Anti-perforin, granzyme, and CD56 were negative (not shown). 
nausea. She was referred for acute kidney injury (AKI), defined by a SCr of $354 \mu \mathrm{moll}^{-1}$ at day 48 . A physical examination excluded hypertension, edema, and skin rash. Laboratory tests identified mild proteinuria (UPCr $0.4 \mathrm{~g} / \mathrm{g}$ ) but no hematuria or leukocyturia. Urinary culture was negative. No extra-renal disorders could be identified. The patient received omeprazole, bromazepam, fluoxetine, levothyroxine for more than 4 years. Screening for Leptospirosis, hepatitis $\mathrm{B}$ and $\mathrm{C}$ viruses, toxocarosis, and HIV was negative. Apart from antinuclear antibodies, which were positive at low levels $(1 / 160)$, other immunological tests were negative or normal (ANCA, anti-glomerular basal-membrane antibodies, complement, serum immunoglobulin including IgG4). Renal ultrasonography was normal. Renal biopsy (day 50) showed severe interstitial nephritis with infiltration of polymorphic inflammatory cells and granulomatosis. The interstitial inflammatory infiltrate was mainly positive for the CD3 and CD4 T-cell marker (Figure 1). Glomeruli were normal except for 2 out of 19, which exhibited severe glomerulosclerosis. Immunostaining for IgA, IgG, IgM, C3, C1q, fibrinogen, and kappa and lambda chains was negative. The patient received oral steroids from days 49 to 78 ( $1 \mathrm{mg} \mathrm{kg}^{-1}$ per day followed by rapid tapering). Nivolumab was definitively withdrawn. On day-220, SCr was $124 \mu \mathrm{moll}^{-1}$ (eGFR $38 \mathrm{ml}-\min ^{-1} / 1.73 \mathrm{~m}^{2}$ ).

Case 2. A 52-year-old woman with $B R A F$ wild-type metastatic melanoma was treated with pembrolizumab as a front-line therapy (basal SCr: $55 \mu \mathrm{moll}^{-1}$ ). On day 137 (18 days after the fourth infusion), she was referred for AKI. Other treatments included pantoprazole, bromazepam, and paracetamol. Three months before AKI, she had herpes zoster and received valaciclovir for 3 weeks. She also had central hypothyroidism with amenorrhea, and diabetes mellitus that started with severe ketoacidosis after the second infusion of pembrolizumab and was related to the treatment. A physical examination was normal. As shown in Figure 1, SCr had increased to $145 \mu \mathrm{moll}^{-1}$. UPCr was $0.3 \mathrm{~g} / \mathrm{g}$. Urinalysis showed neither red nor white cells, and was sterile. Immunological tests were all negative. Kidney biopsy (day 147) showed acute interstitial nephritis with a lymphoplasmacytic cell infiltrate and acute tubular injuries, but no granuloma. Several mucoid and protein casts were identified. Glomeruli exhibited mild mesangial hypertrophy and proliferation. Standard immunostaining was negative. The interstitial inflammatory infiltrate was mainly positive for the CD3 and CD4 T-cell marker (Figure 1). Pembrolizumab was withdrawn and the patient received oral steroids ( $1 \mathrm{mg} \mathrm{kg}^{-1}$ per day followed by progressive decrease). On day 225,15 days after steroid withdrawal, SCr was $85 \mu \mathrm{moll}^{-1}$ (eGFR $65 \mathrm{ml} \mathrm{min}^{-1} / 1.73 \mathrm{~m}^{2}$ ).

Case 3. A 68-year-old woman with BRAF wild-type metastatic melanoma was treated with ipilimumab as a front-line therapy (basal SCr: $99 \mu \mathrm{moll}^{-1}$ ). Other treatments including pantoprazole, amitriptyline chlorhydrate, levothyroxine, and paracetamol were given for several years. By day 130 (87 days after the fourth infusion of ipilimumab), she was referred for AKI ( $\mathrm{SCr}$ $162 \mu \mathrm{moll}^{-1}$, Figure 1). She had developed emesis, rapid weight loss, and diffuse arthralgia. A physical examination revealed diffuse abdominal pain and axillary lymph nodes, but no arthritis. Biological tests identified de novo hypophysitis with central adrenal failure, hyperprolactinemia, and hepatitis. Hypereosinophilia was transiently identified (eosinophil count $2.3 \mathrm{Gl}^{-1}$ ). Patient was negative for anguillulosis, toxocarosis, hydatid cyst, filariasis, and amebiasis. Viral investigation including HIV, parvoB19, hepatitis $\mathrm{B}, \mathrm{C}$, and $\mathrm{E}$ viruses was negative. Immunological tests were all negative. No proteinuria was observed. urinalysis showed neither red nor white cells, and was sterile. Kidney biopsy (day 130) showed mild acute tubular injury and interstitial inflammatory fibrosis (Figure 1). Four out of 30 glomeruli showed severe glomerulosclerosis; all other glomeruli were normal. Standard immunostaining was negative. The interstitial inflammatory infiltrate was mainly positive for the CD3 and CD4 T-cell marker (Figure 1). Ipilimumab was withdrawn and oral steroids were started at a dose of $1 \mathrm{mg} \mathrm{kg}^{-1}$ per day for 1 month. At day 244, SCr was $76 \mu \mathrm{moll}^{-1}$ (eGFR $70 \mathrm{ml} \mathrm{min}{ }^{-1} / 1.73 \mathrm{~m}^{2}$ ).

\section{DISCUSSION}

Herein, we describe three cases of AKI following treatment with ICI. In all patients, kidney biopsy revealed the same tubulointerstitial pattern with infiltration of polymorphic inflammatory cells and predominant $\mathrm{CD} 3+\mathrm{CD} 4+\mathrm{T}$ cells. $\mathrm{PD}-1$ positive and neoantigen-reactive circulating cells have been recently identified in melanoma patients, suggesting that pre-existing clonal populations with autoimmune activity may emerge after exposure to ICI (Gros et al, 2016). Thus, ICI could prime or react with intra-renal $\mathrm{T}$ cells, which subsequently exert local inflammation (Spanou et al, 2006), induce the production of autoantibodies (anti-DNA; Lute et al, 2005) or inhibit T-reg, thereby inducing or enhancing the expansion of auto-reactive $\mathrm{T}$ cells with tropism to the kidneys.

Screening for usual causes of acute interstitial nephritis (AIN; infections, auto-immune diseases) was negative in all patients: all were negative for ENA (Extractable Nuclear Antigens) antibodies including anti-SSA, anti-SSB, SM, SM-RNP, RNP, Scl70, Jo1, centromers, ribosomes; No rhumatoid factor was reported; AntiDNA antibodies, and anti-neutrophil cytoplasmic antibodies were negative for case 1 and 3 (not performed for case 2). The lack of hypercalcemia, chest lymph nodes, and sicca syndrome ruled out sarcoidosis and Sjogren syndrome. No patients had ocular signs, suggesting tubulointerstitial nephritis with uveitis (TINU) syndrome, nor IgG4 elevation (rare causes of AIN).

Among the causes of AIN, drug-related nephritis is the most frequent. The respective roles of ICI and other drugs in the occurrence of AIN need to be clarified. In our patients, no new drug was recently introduced. However, ICI could have released suppression of $\mathrm{T}$-cell immunity that normally permits renal tolerance of drugs known to be associated with AIN, but no experimental data could be used to confirm this hypothesis. Finally, several arguments pointed to ICI: (i) renal disorders improved after cessation of ICI, (ii) chronology between ICI introduction and AKI was more likely than with the other drugs, and (ii) medical literature reported the same effects (Izzedine et al, 2014; Cortazar et al, 2016). AKI is preceded or accompanied by an erythematous skin rash in half of the published cases. In two patients, a syndrome of drug reaction with eosinophilia and systemic symptoms, including AKI and liver-test abnormalities, was diagnosed, which suggests a true allergy to these drugs (Thajudeen et al, 2015) In other patients with a history of or concomitant extra-renal disorders presentation has been more suggestive of an autoimmune disorder linked to the expansion of an autoreactive T-cell clone. Importantly, extra-renal symptoms mostly preceded the onset of nephritis, thus highlighting the need of careful renal monitoring if adverse event is considered nonlimiting and ICI are maintained.

In the Medication Guide provided by the Bristol-Myers Squibb Company (Princeton, NJ 08543 USA), approved by the US Food and Drug Administration, and revised in October 2015, it is recommended to monitor patients for elevated serum creatinine prior to and periodically during treatment with ipilimumab and nivolumab. Creatinine should be monitored at least: once a week if creatinine increases up to 1.5 times above baseline, every 2-3 days if creatinine increases above 1.5 times baseline up to 6 times upper limit of normal, and daily if creatinine increases above 6 times upper limit of normal. To date, no guidelines are available to 
recommend the frequency for the degree of renal monitoring in patients with normal kidney function at drug initiation. Considering the life-threatening potential and severity of acute kidney injury, risk benefit ratio favors early diagnosis. Creatinine measurements should be considered as non-invasive in a context of metastatic cancer, especially when other parameters are often simultaneously assessed (e.g., blood cell counts). Thus, we suggest that creatinine measurement should be performed every 2 weeks for 3-6 months in patients with normal kidney function at drug initiation, especially if they exhibit other immune-related adverse events or if ICI are used in combination.

When comparing ICI themselves, there was no evidence for a ICI-specific clinical pattern. Case 3 (Ipilimumab) showed more extra renal signs and a chronic pattern of interstitial inflammation but the epidemiological data are still lacking to distinguish specific adverse events between nivolumab, pembrolizumab, and ipilimumab.

Renal disorders following ICI are rare. In Toulouse University Hospital, between September 2015 and April 2016, 3 cases of AKI were reported out of 233 patients receiving ICI (168 nivolumab, 45 pembrolizumab, and 20 ipilumumab), thus leading to a global incidence of $1-2 \%$. In a recent study of 752 melanoma patients treated with ipilimumab, 120 adverse events were reported. Among them, only four involved the kidney (Voskens et al, 2013). The time for renal disease to appear varied from 6 to 12 weeks after first exposure to ICI (i.e., after the second infusion). Case reports and a very recent series of 13 patients (totally 28 patients) mostly described acute tubulointerstitial injuries. In one patient, lupus nephritis-related nephrotic syndrome developed after two infusions of ipilimumab (Fadel et al, 2009). One patient developed hypothyroidism or autoimmune-related rhabdomyolysis-induced AKI (Min and Hodi, 2013). One patient also developed nephrotic syndrome with minimal-change disease (Kidd and Gizaw, 2016). Although our study includes only three cases, the number of ICI-induced renal disorders will probably increase along with the widespread use of these molecules. Moreover, because ICI are mostly given to patients along with drugs that have mild-to-moderate-related adverse events, clinicians should be aware of the risk of delayed acute tubulointerstitial nephritis.

The combined literature and our study reveal the following characteristics of ICI-related AKI. Most patients had AKI with tubulointerstitial presentation, including normal urinary output, granular casts (Forde et al, 2012) aseptic leucocyturia, and lowgrade ( $<1 \mathrm{~g}$ per day) or no proteinuria. Microscopic hematuria is rare (Izzedine et al, 2014; Cortazar et al, 2016). Renal size was normal, but imaging (ultrasound or CT) sometimes revealed reversible bilateral swelling of the renal cortices, without dilated pyelocaliceal cavities (Forde et al, 2012). Renal biopsies showed interstitial infiltration of polymorphic inflammatory cells with predominant CD3 + T-cells. Tubular-cell swelling, vacuolization, focal simplification of tubules with bland casts, and regenerative changes have been previously reported (Izzedine et al, 2014). Granulomatous interstitial nephritis has also been described (Thajudeen et al, 2015; Cortazar et al, 2016). The glomeruli were not modified and immunostaining for immunoglobulins and complement was negative.

In most cases, renal recovery occurred after intake of steroids (Thajudeen et al, 2015) and the urinary leukocytes disappeared (Izzedine et al, 2014). However, progression towards renal fibrosis has been the rule, despite the early recognition of AKI, withdrawal of the drug, and the use of corticosteroids. Overall, in our cases, mean eGFR values at the end of follow-up were, respectively, 39, 68 , and $70 \mathrm{ml} \mathrm{min}^{-1} / 1.73 \mathrm{~m}^{2}$ vs 11,36 , and $31 \mathrm{ml} \mathrm{min}^{-1} / 1.73 \mathrm{~m}^{2}$ at presentation. The published data on long-term prognoses, including patients with more severe form (i.e., requiring renal replacement therapy) are lacking.
In summary, all patients receiving ICI should undergo renal monitoring every 2 weeks for 3-6 months. In patients developing AKI, a renal biopsy, drug withdrawal, and corticosteroid therapy $\left(1 \mathrm{mg} \mathrm{kg}^{-1}\right.$ per day during 1 month followed by rapid tapering) could be recommended. The choice of withdrawing or reintroducing ICI should be decided upon after multidisciplinary discussion that includes defining the cancer status and its prognosis, the risk of end-stage renal disease, and also taking the patient's opinion into account. Because a relapse of immune disorders can occur following the use of alternative ICI, patient who have experienced a first immunological flare-up should be closely monitored (Fadel et al, 2009).

\section{CONFLICT OF INTEREST}

The authors declare no conflict of interest.

\section{AUTHOR CONTRIBUTIONS}

JB, DR and SF designed the study and wrote the manuscript; AD performed the renal pathology; All the authors followed the patients; All the authors approved the manuscript.

\section{REFERENCES}

Cortazar FB, Marrone KA, Troxell ML, Ralto KM, Hoenig MP, Brahmer JR, Le DT, Lipson EJ, Glezerman IG, Wolchok J, Cornell LD, Feldman P, Stokes MB, Zapata SA, Hodi FS, Ott PA, Yamashita M, Leaf DE (2016) Clinicopathological features of acute kidney injury associated with immune checkpoint inhibitors. Kidney Int 90: 638-647.

Fadel F, El Karoui K, Knebelmann B (2009) Anti-CTLA4 antibody-induced lupus nephritis. $N$ Engl J Med 361: 211-212.

Forde PM, Rock K, Wilson G, O’Byrne KJ (2012) Ipilimumab-induced immune-related renal failure-a case report. Anticancer Res 32: 4607-4608.

Gros A, Parkhurst MR, Tran E, Pasetto A, Robbins PF, Ilyas S, Prickett TD, Gartner JJ, Crystal JS, Roberts IM, Trebska-McGowan K, Wunderlich JR, Yang JC, Rosenberg SA (2016) Prospective identification of neoantigenspecific lymphocytes in the peripheral blood of melanoma patients. Nat Med 22: 433-438.

Izzedine H, Gueutin V, Gharbi C, Mateus C, Robert C, Routier E, Thomas M, Baumelou A, Rouvier P (2014) Kidney injuries related to ipilimumab. Invest New Drugs 32: 769-773.

Kidd JM, Gizaw AB (2016) Ipilimumab-associated minimal-change disease. Kidney Int 89: 720.

Lute KD, May KF, Lu P, Zhang H, Kocak E, Mosinger B, Wolford C, Phillips G, Caligiuri MA, Zheng P, Liu Y (2005) Human CTLA4 knock-in mice unravel the quantitative link between tumor immunity and autoimmunity induced by anti-CTLA-4 antibodies. Blood 106: 3127-3133.

Min L, Hodi FS (2013) Anti-PD1 Following ipilimumab for mucosal melanoma: durable tumor response associated with severe hypothyroidism and rhabdomyolysis. Cancer Immunol Res 2: 15-18.

Shirali AC, Perazella MA, Gettinger S (2016) Association of acute interstitial nephritis with programmed cell death 1 inhibitor therapy in lung cancer patients. Am J Kidney Dis 68: 287-291.

Spanou Z, Keller M, Britschgi M, Yawalkar N, Fehr T, Neuweiler J, Gugger M, Mohaupt M, Pichler WJ (2006) Involvement of drug-specific T cells in acute drug-induced interstitial nephritis. J Am Soc Nephrol 17: 2919-2927.

Thajudeen B, Madhrira M, Bracamonte E, Cranmer LD (2015) Ipilimumab granulomatous interstitial nephritis. Am J Ther 22: e84-e87.

Topalian SL, Hodi FS, Brahmer JR, Gettinger SN, Smith DC, McDermott DF, Powderly JD, Carvajal RD, Sosman JA, Atkins MB, Leming PD, Spigel DR, Antonia SJ, Horn L, Drake CG, Pardoll DM, Chen L, Sharfman WH, Anders RA, Taube JM, McMiller TL, Xu H, Korman AJ, Jure-Kunkel M, Agrawal S, McDonald D, Kollia GD, Gupta A, Wigginton JM, Sznol M (2012) Safety, activity, and immune correlates of anti-PD-1 antibody in cancer. $N$ Engl J Med 366: 2443-2454. 
Voskens CJ, Goldinger SM, Loquai C, Robert C, Kaehler KC, Berking C, Bergmann T, Bockmeyer CL, Eigentler T, Fluck M, Garbe C, Gutzmer R, Grabbe S, Hauschild A, Hein R, Hundorfean G, Justich A, Keller U, Klein C, Mateus C, Mohr P, Paetzold S, Satzger I, Schadendorf D, Schlaeppi M, Schuler G, Schuler-Thurner B, Trefzer U, Ulrich J, Vaubel J, von Moos R, Weder P, Wilhelm T, Göppner D, Dummer R, Heinzerling LM (2013) The price of tumor control: an analysis of rare side effects of anti-CTLA-4 therapy in metastatic melanoma from the ipilimumab network. PLoS One 8: e53745.

This work is published under the standard license to publish agreement. After 12 months the work will become freely available and the license terms will switch to a Creative Commons AttributionNonCommercial-Share Alike 4.0 Unported License. 